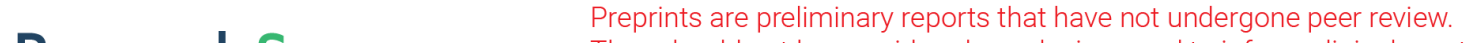 They should not be considered conclusive, used to inform clinical practice, or referenced by the media as validated information. \\ Evolution of Distributed Detection Performance over Balanced Binary Relay Tree Networks with Bit Errors
}

\section{Yu-Ping Tian ( $\nabla$ yptian@hdu.edu.cn )}

Hangzhou Dianzi University https://orcid.org/0000-0003-1193-3394

\section{Wenbo Zhu}

Southeast University

\section{Research Article}

Keywords: Distributed detection, Distributed hypothesis testing, Nonlinear switched system, Invariant set, Channel noise

Posted Date: June 17th, 2021

DOl: https://doi.org/10.21203/rs.3.rs-259853/v1

License: (1) This work is licensed under a Creative Commons Attribution 4.0 International License. Read Full License

Version of Record: A version of this preprint was published at Nonlinear Dynamics on January 17th, 2022. See the published version at https://doi.org/10.1007/s11071-021-06736-1. 


\title{
Evolution of Distributed Detection Performance over Balanced Binary Relay Tree Networks with Bit Errors
}

\author{
Yu-Ping Tian · Wenbo Zhu
}

Received: date / Accepted: date

\begin{abstract}
Target detection based on wireless sensor networks can be considered as a distributed binary hypothesis testing problem. In this paper, the evolution of detection error probability with the increase of network scale is studied for the balanced binary relay tree network with channel noise. Firstly, the iterative expressions of false-alarm probability and missed-detection probability depending on the number of tree network layers are given. Then, the iterative process of two types of error probabilities in the network space is described as a discrete nonlinear switched dynamic system, and the dynamic properties of two types of error probabilities are analyzed in a plane rectangular coordinate system. A globally attractive invariant set of the state of the dynamic system, which is not related to the channel noise, is derived. The switching mode of the system and the total error probability in the invariant set are further analyzed, and a necessary and sufficient convergence condition of the total error probability is provided. Based on this condition the following detection properties of the network are revealed: (1) as long as the channel bit error probability is not zero, the total error probability does not tend to zero with the increasing network size; (2) when the channel bit error probability is greater than $\frac{2-\sqrt{3}}{2}$, the total error probability will continue to increase with the increase of network size.
\end{abstract}

Keywords Distributed detection - Distributed hypothesis testing · Nonlinear switched system · Invariant set $\cdot$ Channel noise

\section{Introduction}

Target detection based on wireless sensor networks (WSNs) has a very wide and important application background and has received constant attention from industry

Yu-Ping Tian

School of Automation, Hangzhou Dianzi University, Hangzhou, 310018, China.

E-mail: yptian@hdu.edu.cn (Corresponding author)

Wenbo Zhu

School of Automation, Southeast University, Nanjing, 210096, China. 
and academia [1-4]. In particular, the relationship between distributed detection performance and sensor network size has been studied in detail for different network structures. Parallel structure is the first to receive attention. In the network of this structure, each sensor directly submits its detection results to the fusion center (FC). When each sensor has independent observations, the parallel structure can ensure that the total error probability of FC decreases exponentially with the increase of network size [5]. However, when the physical distance between a sensor and FC is far away, the parallel structure is not conducive to energy saving, and is usually difficult to implement in the case of large scale networks. An alternative structure is tandem network [6-8]. In tandem networks, each node fuses its own detection results with the detection results of lower-level nodes, and then transmits the fusion results to an upper-level node. Under this structure, the decision-making error probability at FC decreases sub-exponentially with the increase of network size [8]. Tree network is something between parallel structure and tandem structure. In this kind of network, in addition to the FC and the leaf nodes which directly detects the target, there are also a number of relay nodes which are only responsible for local information fusion and transmission. Tree networks have attracted more and more attention because they can take into account both the energy consumption and detection performance in practical applications.

For the tree networks with finite tree height (i.e., when the network size tends to infinity, the tree height is finite), paper [9] proves that under Bayesian criterion the error probability of FC exponentially decays with the increase of network size although the decay exponent is smaller than that of parallel network. Paper [10] studies a kind of tree network with infinite tree height (i.e., when the network size tends to infinity, the tree height also tends to infinity), the so-called balanced binary relay tree (BBRT) network. Under the premise that all leaf-nodes' observations are independent and al1 relay nodes adopt the same likelihood ratio test (LRT), it is proved by Lyapunov method that the total error probability of FC converges to zero. In [11], the derivation of optimal fusion rules in the BBRT network is investigated by using dynamic programming method. Under the unit threshold LRT (UT-LRT) rule, paper [12] studies the binary hypothesis testing in the BBRT network, analyzes the dynamical properties of false-alarm probability (probability of Type I error) and missed-detection probability (probability of Type II error), and derives a globally attractive invariant set in the state space of error probabilities of two types. Based on the dynamic property analysis in this invariant set, it is proved that the decay exponent of the total error probability of the network is the square root of the number of network layers.

All the above-mentioned researches are aimed at dealing with ideal networks. In practical applications, however, WSNs are often deployed in complex environments. Many environmental factors may interfere with the communication between sensor nodes, which will reduce the stability and accuracy of communication to a certain extent. More and more attention has been paid to the distributed detection performance of WSNs with network failure or communication noise. For the problem of network failure, paper [13] introduces node and link failure probabilities, and studies the asymptotic detection performance of the BBRT network under the UT-LRT criterion. In paper [14], the asymptotic detection performance of the M-ary tree network with node and link failures is studied under the majority dominance rule. The upper 
and lower bounds of the convergence rate of the FC's error probability with respect to the network size are also given. Both [13] and [14] show that the existence of node and link failures with a certain probability does not overthrow the fact that the error probability at FC decreases to zero as the network size grows to infinity. Besides, the influence of Gaussian noise in communication channels on the distributed detection performance of WSNs has also been extensively investigated in the literature [15-17].

The bit error caused by communication noise in binary communication channel has a completely different impact on communication from network failure, and it can change the transferred information from "true" to "false". Binary symmetric channels (BSC) is a widely-used model to describe noisy binary channels. Paper [18] studies the design of parameter estimator with binary quantitative observations under BSC model. Paper [19] compares the advantages and disadvantages of various information fusion rules in BSC model. A distributed detection method based on Rao test is studied for noisy channels in [20]. Paper [21] considers the case that the bit noise exists in the communication between sensor node and FC, and constructs an extended maximum likelihood estimator. However, there are few relevant reports concerning the questions such as: How does the FC's error probability evolve in WSNs with bit errors with respect to the network size? Will it decay to zero as the networks size grows to infinity? Recently, the authors' group has investigated these questions for the M-ary tree network and has given a negative answer to the second question [22].

In this paper, we consider the distributed detection problem in the BBRT network with bit error communication channels, and analyze the asymptotic detection performance of the network under BSC model. Firstly, we derive the iterative expression of false-alarm probability and missed-detection probability with respect to the number of tree network layers, and model the iterative process of the two kinds of error probabilities as a state-dependent nonlinear switched dynamic system. Comparing the dynamic model of the BBRT model derived in this paper and the model of the M-ary tree network given in [22], we find that the former is much more complicated because it is a switched system besides nonlinearity while the latter is just a nonlinear stationary system. Afterwards, since it is difficult to make the equilibrium analysis like [22] for this nonlinear switched system, we analyze the dynamic properties of the system by dividing the state space as mode-holding regions and mode-switching regions. Based on the state space partitioning, a globally attractive invariant set of the state of the dynamic system is obtained. Finally, we study the switching mode of the dynamic system in the globally attractive invariant set, and give a necessary and sufficient condition for the convergence of the total error probability of FC. It is pointed out that the critical value of the bit error probability that makes the total error probability unable to converge is $\frac{2-\sqrt{3}}{2}$. This critical value is meaningful for the design of large-scale wireless sensor networks.

The research method of this paper is greatly inspired by paper [13]. However, unlike the node and link failure discussed in [13], the BSC channel discussed in this paper will produce a signal with the opposite meaning to the source signal. The introduction of bit error probability in the dynamic model makes the evolution process of detection error probability much more complex, and the derivation and proof of the invariant set become much more difficult. And the result obtained in this paper is also quite different from that of [13]: the conclusion that the total error probability 
will decay to zero with the infinite growth of network size is no longer true for the BSC model.

The following notations are used in this note. $P(\cdot)$ denotes probability. $\alpha_{k}, \beta_{k}$ and $d_{k}=1-\beta_{k}$ denote the false-alarm probability, missed-detection probability and detection probability of a node at level $k$ in a BBRT network, respectively. The channel bit-error probability is denoted by $b$. And the closure of set $A$ is denoted by $\bar{A}$.

The rest of this paper is organized as follows. The problem formulation and dynamic model of detection performance over BBRT is presented in Section 2. In Section 3, we study the evolution of the detection error probabilities of two types by dividing their state space into different regions. In Section 4 , a channel-noiseindependent and globally attractive invariant set of the detection error probabilities is constructed. The switching mode of the dynamic system in the invariant set is also studied in this section. The asymptotic property of the total error probability is analyzed in Section 5. The conclusion is drawn in Section 6.

\section{Dynamic Model of Detection Performance of UT-LRT in BBRT with Noise}

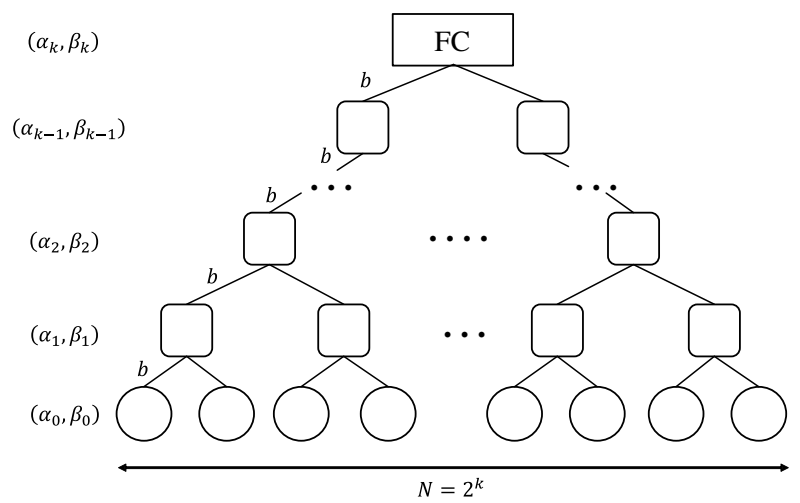

Fig. 1 Architecture of balanced binary relay tree with channel noise.

We consider the target detection as a binary hypothesis testing problem: hypothesis $H_{1}$ corresponds to the appearance of the target and hypothesis $H_{0}$ corresponds to the absence of the target. Let such a detection be conducted in a BBRT network with channel noise as shown in Fig. 1, where circles represent sensors, also called leaf nodes, which make measurements, squares represent relay nodes which only fuse messages, rectangle represents the fusion center which makes an overall decision, $k$ denotes the layer number of the tree, $N$ is the total number of sensors. Each leaf node makes independent detections for the same events, then summarizes it into binary message and sends the message to its parent node. Each parent node receives message sent by two child nodes, then it will fuse two binary messages into one bi- 
nary message and forward the new binary message to its parent node. This process is repeating until the message is transmitted to the root node, which is the fusion center.

The channel noise modeled by BSC is shown in Fig. 2. The bit-error probability of the BSC is denoted by $b$. When a child node sends $X$ to its parent node, the parent node receives $1-X$ with probability $b$ and receives $X$ with probability $1-b$.

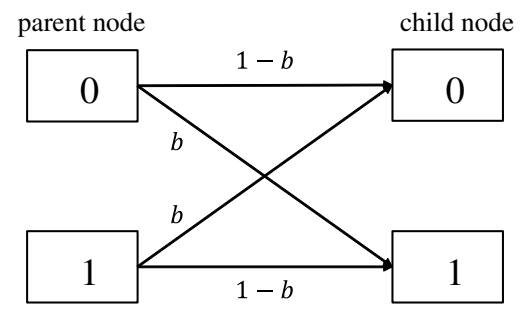

Fig. 2 Binary symmetric channel noise.

The following assumptions are needed for our further study:

Assumption 1 All sensors are independent with identical false-alarm probability $\alpha_{0}$ and identical missed-detection probability $\beta_{0}$, which are subject to $\alpha_{0}+\beta_{0}<1$.

Assumption 2 All channels in the network have the same bit-error probability $b \in$ $\left[0, \frac{1}{2}\right)$.

The part of Assumption 1 that all sensors are independent with identical falsealarm probability and identical missed-detection probability is made for the possibility of iterative analysis of the BBRT network (see, e.g. [12,13]). For the BSC model, the assumption $\alpha_{0}+\beta_{0}<1$ and Assumption 2 do not reduce the generality of the results of this paper because the case of $\alpha_{0}+\beta_{0}>1$ or the case $b \in\left(\frac{1}{2}, 1\right]$ can be considered as a folded case: if we redefine the meaning of the binary decision 0 as 1 and 1 as 0 , then we will return to the case $\alpha_{0}+\beta_{0}<1$ or the case $b \in\left[0, \frac{1}{2}\right)$.

Suppose that the UT-LRT rule is applied at each relay node and the fusion center. It has been shown that this fusion rule is locally optimal if the event detected has equal priori probability, i.e., $P\left(H_{0}\right)=P\left(H_{1}\right)=\frac{1}{2}$ (see, e.g.,[10]).

Now, let us define $F(b, x):[0,0.5] \times[0,1] \rightarrow[0,1]$ as

$$
\begin{aligned}
F(b, x) & =(1-b) x+b(1-x) \\
& =(1-2 b) x+b .
\end{aligned}
$$

Considering $x$ as the Type I (or Type II) error probability of a decision, then $F(b, x)$ is actually the Type I (or type II) probability of the decision signal passessing through the BSC channel with bit error probability $b$. Apparently, for any $x \in[0,1]$ and $y \in$ $[0,1]$, the following equivalence holds

$$
\begin{aligned}
& x \leq y \Leftrightarrow F(b, x) \leq F(b, y), \\
& x+y \leq 1 \Leftrightarrow F(b, x)+F(b, y) \leq 1 .
\end{aligned}
$$


Theorem 1 Under Assumptions 1, 2 and the UT-LRT rule, the relation of the falsealarm probability and missed-detection probability between two consecutive layers of the BBRT network with bit-error can be described by

$$
\left(\alpha_{k+1}, \beta_{k+1}\right)= \begin{cases}\left(1-\left(1-F\left(b, \alpha_{k}\right)\right)^{2},\left(F\left(b, \beta_{k}\right)\right)^{2}\right), & \text { if } \alpha_{k} \leq \beta_{k} \\ \left(\left(F\left(b, \alpha_{k}\right)\right)^{2}, 1-\left(1-F\left(b, \beta_{k}\right)\right)^{2}\right), & \text { if } \alpha_{k}>\beta_{k},\end{cases}
$$

where $k \geq 0$ denotes the layer of the tree.

Proof At first, let us analyze a local network which consists of a parent node and two child nodes, both child nodes are leaves. By Assumption 1, the leaf nodes have identical false-alarm probability $\alpha_{0}$ and identical detection probability $d_{0}=1-\beta_{0}$. We denote by $X$ the message that the parent node receives from one child node, $Y$ the message from the other child node, and let $Z=(X, Y)$. By Assumption 1 on the independence of the leaf nodes and Assumption 2 on the bit-error probability, it is easy to get:

$$
\begin{aligned}
& P\left(X=1 \mid H_{0}\right)=P\left(Y=1 \mid H_{0}\right)=F\left(b, \alpha_{0}\right), \\
& P\left(X=0 \mid H_{0}\right)=P\left(Y=0 \mid H_{0}\right)=1-F\left(b, \alpha_{0}\right), \\
& P\left(X=1 \mid H_{1}\right)=P\left(Y=1 \mid H_{1}\right)=F\left(b, d_{0}\right), \\
& P\left(X=0 \mid H_{1}\right)=P\left(Y=0 \mid H_{1}\right)=1-F\left(b, d_{0}\right) .
\end{aligned}
$$

Denote by $D$ the decision made by the parent node based on the observation $Z$ and the UT-LRT rule. By the meaning of false-alarm probability and miss-detection probability, we know that $P\left(D=1 \mid H_{0}\right)=\alpha_{1}, P\left(D=1 \mid H_{1}\right)=1-\beta_{1}=d_{1}$. Now, we investigate the conditions under which the decision $D=1$ can be taken based on the UT-LRT rule. There are totally four possible observations as listed below.

Case 1: $Z=(0,0)$. In this case the UT-LRT rule implies that

$$
\frac{P\left(X=0 \mid H_{1}\right) P\left(Y=0 \mid H_{1}\right)}{P\left(X=0 \mid H_{0}\right) P\left(Y=0 \mid H_{0}\right)}=\frac{\left(1-F\left(b, d_{0}\right)\right)^{2}}{\left(1-F\left(b, \alpha_{0}\right)\right)^{2}} \geq 1 .
$$

That is, in this case $D=1$ if $F\left(b, d_{0}\right) \leq F\left(b, \alpha_{0}\right)$. In consideration of $F\left(b, d_{0}\right)=$ $1-F\left(b, \beta_{0}\right)$ and Eq. (2), $F\left(b, d_{0}\right) \leq F\left(b, \alpha_{0}\right)$ is equivalent to $\alpha_{0}+\beta_{0} \geq 1$, which contradicts Assumption 1. This implies that when $Z=(0,0)$, the decision $D=1$ can never be taken under UT-LRT.

Case 2: $Z=(1,0)$. In this case the UT-LRT rule implies that

$$
\frac{P\left(X=1 \mid H_{1}\right) P\left(Y=0 \mid H_{1}\right)}{P\left(X=1 \mid H_{0}\right) P\left(Y=0 \mid H_{0}\right)}=\frac{F\left(b, d_{0}\right)\left(1-F\left(b, d_{0}\right)\right)}{F\left(b, \alpha_{0}\right)\left(1-F\left(b, \alpha_{0}\right)\right)} \geq 1 .
$$

That is, in this case $D=1$ if $F\left(b, d_{0}\right)+F\left(b, \alpha_{0}\right) \leq 1$. In consideration of $F\left(b, d_{0}\right)=$ $1-F\left(b, \beta_{0}\right)$ and Eq. (2), we know $F\left(b, d_{0}\right)+F\left(b, \alpha_{0}\right) \leq 1$ is equivalent to $\alpha_{0} \leq \beta_{0}$.

Case 3: $Z=(0,1)$. It is easy to see that this case is equivalent to the case that $Z=(1,0)$. Hence, $D=1$ if $\alpha_{0} \leq \beta_{0}$.

Case $4: Z=(1,1)$. In this case the UT-LRT rule implies that

$$
\frac{P\left(X=1 \mid H_{1}\right) P\left(Y=1 \mid H_{1}\right)}{P\left(X=1 \mid H_{0}\right) P\left(Y=1 \mid H_{0}\right)}=\frac{\left(F\left(b, d_{0}\right)\right)^{2}}{\left(F\left(b, \alpha_{0}\right)\right)^{2}} \geq 1 .
$$


That is, in this case $D=1$ if $F\left(b, d_{0}\right) \geq F\left(b, \alpha_{0}\right)$. In consideration of $F\left(b, d_{0}\right)=$ $1-F\left(b, \beta_{0}\right)$ and Eq. (3), we know $F\left(b, d_{0}\right) \geq F\left(b, \alpha_{0}\right)$ is equivalent to $\alpha_{0}+\beta_{0} \leq 1$. This implies that when $Z=(1,1)$, it always takes the decision $D=1$ under UT-LRT.

Summarizing the above four cases, we know that $P(D=1)=P(\{Z=(1,0), Z=$ $(0,1), Z=(1,1)\})=1-P(\{Z=(0,0)\})$ if $\alpha_{0} \leq \beta_{0}$, and $P(D=1)=P(\{Z=(1,1)\})$ if $\alpha_{0}>\beta_{0}$. Therefore,

$$
\begin{aligned}
\alpha_{1} & =P\left(D=1 \mid H_{0}\right) \\
& =1-P\left(\{Z=(0,0)\} \mid H_{0}\right) \\
& =1-\left(1-F\left(b, \alpha_{0}\right)\right)^{2}
\end{aligned}
$$

for the case $\alpha_{0} \leq \beta_{0}$, and

$$
\begin{aligned}
\alpha_{1} & =P\left(D=1 \mid H_{0}\right) \\
& =P\left(\{Z=(1,1)\} \mid H_{0}\right) \\
& =\left(F\left(b, \alpha_{0}\right)\right)^{2}
\end{aligned}
$$

for the case $\alpha_{0}>\beta_{0}$. Similarly, since $\beta_{1}=1-d_{1}=1-P\left(D=1 \mid H_{1}\right)$, we have

$$
\begin{aligned}
\beta_{1} & =1-\left(1-P\left(\{Z=(0,0)\} \mid H_{1}\right)\right) \\
& =1-\left(1-\left(1-F\left(b, d_{0}\right)\right)^{2}\right) \\
& =\left(F\left(b, \beta_{0}\right)\right)^{2}
\end{aligned}
$$

for the case $\alpha_{0} \leq \beta_{0}$, and

$$
\begin{aligned}
\beta_{1} & =1-P\left(\{Z=(1,1)\} \mid H_{1}\right) \\
& =1-\left(F\left(b, d_{0}\right)\right)^{2} \\
& =1-\left(1-F\left(b, \beta_{0}\right)\right)^{2}
\end{aligned}
$$

for the case $\alpha_{0}>\beta_{0}$.

Thus, we have proved Eq. (4) in Theorem 1 holds for $k=0$. This equation also shows that all nodes at level 1 have identical false-alarm probability $\alpha_{1}$ and identical missed-detection probability $\beta_{1}$. Moreover, by Eqs (2),(3) and Eq. (4), it follows from $\alpha_{0}+\beta_{0}<1$ that $\alpha_{1}+\beta_{1}<1$ also holds. Since the UT-LRT rule is applied to all nodes in the tree, and all channels in the tree have the same bit-error probability, one can repeat the above proof procedure from $k=1$ to any positive integer and obtain the conclusion that the Eq. (4) holds for all $k \geq 0$.

The iterative equation (4) can be regarded as a discrete dynamic system, which determines how the false-alarm probability $\alpha_{k}$ and the missed-detection probability $\beta_{k}$ evolve in the BBRT with respect to the number of the tree level $k$. Obviously, it is a state-dependent nonlinear switched system. Paper [12] has studied this system for a special case that $b=0$, i.e., the case of no bit-error. An invariant set for the system state has been constructed, and with the help of the analysis of the system dynamics in this invariant set it has been shown that both the false-alarm probability and missed-detection probability converge to zero as the number of sensor in the network increases to infinity. However, in this paper we will show that neither the false-alarm 
probability nor missed-detection probability converge to zero in the case that $b \neq 0$, and the system has very rich and complicated nonlinear dynamic properties. In the following sections we will study the relationship between the convergence property of the system and the channel parameter $b$ via dividing the state space according to the iterative mode of the system.

\section{Evolution of Detection Error Probabilities}

\subsection{Mode-switching region in state space}

As mentioned in the proof of Theorem $1, \alpha_{0}+\beta_{0}<1$ implies that $\alpha_{1}+\beta_{1}<1$ for all $k \geq 0$. The state space of the dynamic system (4) is a right triangle in the rectangular coordinate system denoted by $\sum(\alpha, \beta)$, which consists of upper part $U$ and lower part $L$ given below

$$
\begin{aligned}
U & :=\{(\alpha, \beta) \mid \alpha \geq 0, \beta \geq 0, \beta \geq \alpha, \alpha+\beta<1\}, \\
L & :=\{(\alpha, \beta) \mid \alpha \geq 0, \beta \geq 0, \beta<\alpha, \alpha+\beta<1\} .
\end{aligned}
$$

We note that Eq. (4) is symmetric about the line $\alpha=\beta$. Hence, if a dynamic property of system (4) holds in region $U$, it must also hold in a symmetric way in region $L$. We refer to this as dynamic symmetry of system (4), and use it to shorten the length of the proof of most conclusions in this paper.

The iteration of system (4) switches between two modes

$$
\begin{aligned}
& f_{1}\left(\alpha_{k}, \beta_{k}\right):=\left(1-\left(1-F\left(b, \alpha_{k}\right)\right)^{2},\left(F\left(b, \beta_{k}\right)\right)^{2}\right), \\
& f_{2}\left(\alpha_{k}, \beta_{k}\right):=\left(\left(F\left(b, \alpha_{k}\right)\right)^{2}, 1-\left(1-F\left(b, \beta_{k}\right)\right)^{2}\right)
\end{aligned}
$$

depending on whether the state of the system falls in $L$ or $U$ at each step. To study the switching mode of the system we define the mode-switching region of the dynamic system (4) as follows.

Definition 1 A set $A_{s}$ in $\sum(\alpha, \beta)$ is referred to as a mode-switching region of dynamic system (4) iffor all $\left(\alpha_{k}, \beta_{k}\right) \in A_{s}$, both $\left(\alpha_{k+1}, \beta_{k+1}\right)=f_{i}\left(\alpha_{k}, \beta_{k}\right)$ and $\left(\alpha_{k+2}, \beta_{k+2}\right)=$ $f_{j}\left(\alpha_{k+1}, \beta_{k+1}\right)$ hold, where $i, j \in\{1,2\}, i \neq j$.

Define

$$
\begin{aligned}
B_{1 U} & :=\left\{(\alpha, \beta) \in U \mid(1-F(b, \alpha))^{2}+(F(b, \beta))^{2}<1\right\}, \\
B_{1 L} & :=\left\{(\alpha, \beta) \in L \mid(1-F(b, \beta))^{2}+(F(b, \alpha))^{2} \leq 1\right\} .
\end{aligned}
$$

Apparently, $\overline{B_{1 U}}$ and $\overline{B_{1 L}}$ are symmetric with respect to the line $\beta=\alpha$.

Proposition 1 Consider system (4). If $\left(\alpha_{k}, \beta_{k}\right) \in B_{1 U}$, then $\left(\alpha_{k+1}, \beta_{k+1}\right) \in L$; if $\left(\alpha_{k}, \beta_{k}\right) \in$ $B_{1 L}$, then $\left(\alpha_{k+1}, \beta_{k+1}\right) \in U$.

Proof Let $\left(\alpha_{k}, \beta_{k}\right) \in B_{1 U}$, i.e., $\left(\alpha_{k}, \beta_{k}\right) \in U$ and $\left(1-F\left(b, \alpha_{k}\right)\right)^{2}+\left(F\left(b, \beta_{k}\right)\right)^{2}<1$. Then, it follows from (4) that $\alpha_{k+1}=1-\left(1-F\left(b, \alpha_{k}\right)\right)^{2}>\left(F\left(b, \beta_{k}\right)\right)^{2}=\beta_{k+1}$, i.e., $\left(\alpha_{k+1}, \beta_{k+1}\right) \in L$. According to the dynamic symmetry of the system, it is also true that $\left(\alpha_{k+1}, \beta_{k+1}\right) \in U$ if $\left(\alpha_{k}, \beta_{k}\right) \in B_{1 L}$. 


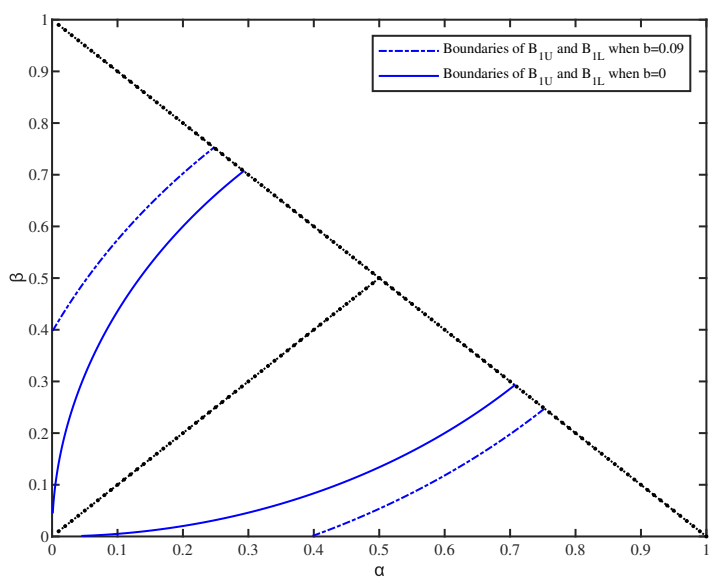

Fig. 3 Region $B_{1}$ for $b=0.09$ and $b=0$.

Proposition 1 says that $B_{1}:=B_{1 U} \cup B_{1 L}$ is a mode-switching region of system (4).

The following proposition reveals the relationship of the mode-switching regions of the system in the noisy channel case and noise-free case.

Proposition $\left.\left.2 B_{1}\right|_{b=0} \subset B_{1}\right|_{b \neq 0}$.

Proof of Proposition 2 is given in Appendix A.

Proposition 2 is illustrated by Fig. 3, which shows that the upper boundary of $B_{1 U}$ in the case of $b \neq 0$ is higher than the upper boundary of $B_{1 U}$ in the case of $b=0$. Symmetrically, the lower boundary of $B_{1 L}$ in the case of $b \neq 0$ is lower than the lower boundary of $B_{1 L}$ in the case of $b=0$.

3.2 Mode-holding region in state space

Corresponding to mode-switching region, mode-holding region is defined as follows.

Definition $2 A$ set $A_{h}$ in $\sum(\alpha, \beta)$ is referred to as a mode-holding region of dynamic system (4) if for all $\left(\alpha_{k}, \beta_{k}\right) \in A_{h}$, both $\left(\alpha_{k+1}, \beta_{k+1}\right)=f_{i}\left(\alpha_{k}, \beta_{k}\right)$ and $\left(\alpha_{k+2}, \beta_{k+2}\right)=$ $f_{i}\left(\alpha_{k+1}, \beta_{k+1}\right)$ hold, where $i \in\{1,2\}$.

For convenience of discussion, let us define a function $D(b, x):[0,0.5] \times[0,1] \rightarrow$ $[0,1]$ as

$$
D(b, x)=(1-2 b) x^{2}+b,
$$

and denote the $m$-th composition of this function as $D^{m}(b, x)$, i.e.,

$$
D^{m}(b, x)=D \overbrace{(b, \cdots, D(b, D(b, x)) \cdots}^{m \text { times }} .
$$


And then, we further divide the state space of system (4) as follows.

$$
\begin{aligned}
B_{2 U} & :=\left\{(\alpha, \beta) \in U \backslash B_{1 U} \mid D(b,(1-F(b, \alpha))+D(b, F(b, \beta))<1\},\right. \\
B_{2 L} & :=\left\{(\alpha, \beta) \in L \backslash B_{1 L} \mid D(b,(1-F(b, \beta))+D(b, F(b, \alpha)) \leq 1\},\right. \\
& \ldots \\
& \ldots \\
B_{m U} & :=\left\{(\alpha, \beta) \in U \backslash \bigcup_{i=1}^{m-1} B_{i U} \mid D^{m-1}\left(b,(1-F(b, \alpha))+D^{m-1}(b, F(b, \beta))<1\right\},\right. \\
B_{m L} & :=\left\{(\alpha, \beta) \in L \backslash \bigcup_{i=1}^{m-1} B_{i L} \mid D^{m-1}\left(b,(1-F(b, \beta))+D^{m-1}(b, F(b, \alpha)) \leq 1\right\},\right. \\
& \ldots
\end{aligned}
$$

where $F(b, *)$ is defined by (1). Apparently, $\overline{B_{m U}}$ and $\overline{B_{m L}}$ are symmetric about line $\beta=\alpha$.

Proposition 3 Consider dynamic system (4). If $\left(\alpha_{k}, \beta_{k}\right) \in B_{m U}$, then $\left(\alpha_{k+1}, \beta_{k+1}\right) \in$ $B_{(m-1) U}$; if $\left(\alpha_{k}, \beta_{k}\right) \in B_{m L}$, then $\left(\alpha_{k+1}, \beta_{k+1}\right) \in B_{(m-1) L}$, where $m \geq 2$.

Proof of Proposition 3 is given in Appendix B.

According to Proposition 3, if the initial state $\left(\alpha_{0}, \beta_{0}\right) \in B_{m U}$, where $m \geq 2$, the iterative trajectory of $\left(\alpha_{k}, \beta_{k}\right)$ in the plane of $\sum(\alpha, \beta)$ is subject to the order: $B_{m U} \rightarrow B_{(m-1) U} \rightarrow \cdots B_{2 U} \rightarrow B_{1 U}$; similarly, if $\left(\alpha_{0}, \beta_{0}\right) \in B_{m L}$, where $m \geq 2$, the iterative trajectory of $\left(\alpha_{k}, \beta_{k}\right)$ is subject to the order: $B_{m L} \rightarrow B_{(m-1) L} \rightarrow \cdots B_{2 L} \rightarrow B_{1 L}$. Therefore, the regions $B_{m}, m \geq 2$, are mode-holding regions because the iterations from $B_{m U}$ to $B_{1 U}$ or the iterations from $B_{m L}$ to $B_{1 L}$ share a same iteration mode $f_{1}$ or $f_{2}$.

\subsection{Evolution in the whole state space}

We have shown that $B_{1}$ is a mode-switching region, $B_{m}:=B_{m U} \cup B_{m L}$ are modeholding regions for all $m \geq 2$, in other words, $\cdots B_{m} \cup B_{(m-1)} \cup \cdots \cup B_{2}$ can be considered as a mode-holding region composed of the upper and lower parts. In the state space of the system, is there any other area outside $\cup_{m=1}^{\infty} B_{m}$ ? The following proposition gives a negative answer to this question.

Proposition $4 U \cup L \subseteq \cup_{m=1}^{\infty} B_{m}$.

Proof of the Proposition 4 is given in Appendix C.

Proposition 4 says $\cup_{m=1}^{\infty} B_{m}$ covers the whole state space $U \cup L$. Therefore, by Proposition 3, all the areas outside $B_{1}$ in the state space is the mode-holding region, and the following corollary is obvious.

Corollary 1 For any initial state $\left(\alpha_{0}, \beta_{0}\right) \in U \cup L$, there must exists a positive integer $k^{*}$ such that the trajectory of system (4) starting from $\left(\alpha_{0}, \beta_{0}\right)$ must enter the region $B_{1}$ after $k^{*}$-step iteration, i.e., $\left(\alpha_{k^{*}}, \beta_{k^{*}}\right) \in B_{1}$. 


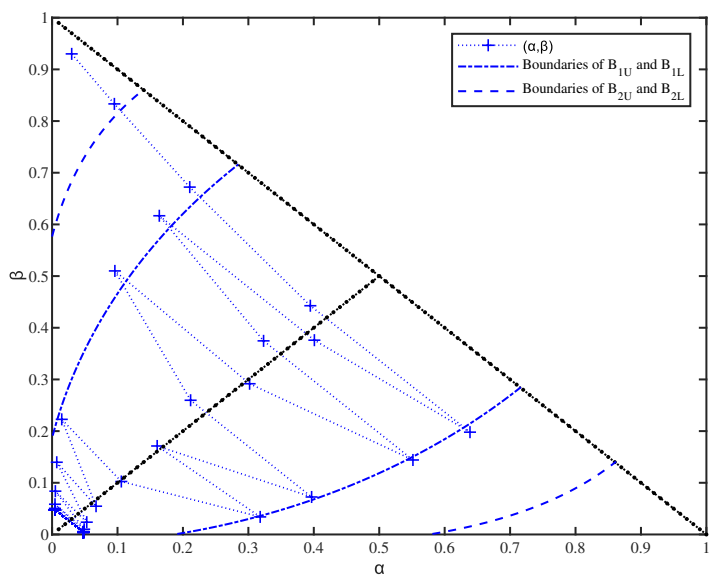

Fig. 4 Iteration trajectory passing through regions $B_{1}$ and $B_{2}$.

Fig. 4 presents a simulation example of the iterative trajectory when $\left(\alpha_{0}, \beta_{0}\right) \in$ $U \backslash\left(B_{1 U} \cup B_{2 U}\right)$. From the trajectory we see that before it enters $B_{1}$ there is no area switching from $U$ to $L$ or from $L$ to $U$, which implies that there is no iteration mode switching between $f_{1}$ and $f_{2}$. Once it enters $B_{1}$, at the next state it must jump from $U$ to $L$ or from $L$ to $U$, which implies that the iteration mode switches from $f_{1}$ to $f_{2}$ or from $f_{2}$ to $f_{1}$. However, the results we have obtained up to now do not tell us into which region it will falls after such a jump, mode-switching region or mode-holding region. This problem is left for the next section.

\section{Globally Attractive Invariant Set}

In this section we investigate the dynamic properties of system (4) after its trajectory enters the region $B_{1}$.

Firstly, let us define

$$
\begin{aligned}
& R_{U}:=\{(\alpha, \beta) \in U \mid \sqrt{1-\beta}+\sqrt{\alpha}>1\}, \\
& R_{L}:=\{(\alpha, \beta) \in L \mid \sqrt{1-\alpha}+\sqrt{\beta} \geq 1\},
\end{aligned}
$$

and denote $R=R_{U} \cup R_{L}$.

Proposition 5 Consider dynamic system (4). If $\left(\alpha_{k}, \beta_{k}\right) \in B_{1 U}$, then $\left(\alpha_{k+1}, \beta_{k+1}\right) \in$ $R_{L}$; if $\left(\alpha_{k}, \beta_{k}\right) \in B_{1 L}$, then $\left(\alpha_{k+1}, \beta_{k+1}\right) \in R_{U}$.

Proof Firstly, suppose that $\left(\alpha_{k}, \beta_{k}\right) \in L$, i.e., $\alpha_{k}>\beta_{k}$, which implies $F\left(b, \alpha_{k}\right)>$ $F\left(b, \beta_{k}\right)$ by (2). By (4) we have $F\left(b, \alpha_{k}\right)=\sqrt{\alpha_{k+1}}, F\left(b, \beta_{k}\right)=1-\sqrt{1-\beta_{k+1}}$, which implies that if $\left(\alpha_{k}, \beta_{k}\right) \in L$ then $\sqrt{\alpha_{k+1}}+\sqrt{1-\beta_{k+1}}>1$. Since $\left(\alpha_{k}, \beta_{k}\right) \in B_{1 L}$, according to Proposition $1,\left(\alpha_{k+1}, \beta_{k+1}\right) \in\{(\alpha, \beta) \in U \mid \sqrt{1-\beta}+\sqrt{\alpha}>1\}$.

The case of $\left(\alpha_{k}, \beta_{k}\right) \in B_{1 U}$ can be proved similarly. 
Proposition $6 R \subset B_{1} \cup B_{2}$.

Proof of Proposition 6 is given Appendix D.

Proposition 7 The upper (lower) boundary of $B_{1}$ and the upper (lower) boundary of $R$ have no intersection if $b \in\left[\frac{3-2 \sqrt{2}}{2}, \frac{1}{2}\right)$; and they have only one intersection point if $b \in\left[0, \frac{3-2 \sqrt{2}}{2}\right)$.

Proof The upper boundary equations of $B_{1 U}$ and $R_{U}$ are

$$
(1-F(b, \alpha))^{2}+(F(b, \beta))^{2}=1
$$

and

$$
\sqrt{1-\beta}+\sqrt{\alpha}=1
$$

respectively. Observing these equations we find that the upper boundary of $R_{U}$ goes through the origin of the coordinate system, but the upper boundary of $B_{1 U}$ does not when $b \neq 0$.

Combining Eqs (7) and (8) yields

$$
(1-2 b)^{2} \alpha^{2}-2(1-2 b)^{2} \alpha \sqrt{\alpha}+(1-2 b)(1-4 b) \alpha+2 b(1-2 b) \sqrt{\alpha}+b^{2}-b=0 .
$$

It can be considered as a quartic equation of $t=\sqrt{\alpha}$. Note the admissible value range of $\alpha$ is $\left[0, \frac{1}{4}\right)$ because the abscissa of the intersection of the upper boundary of $R_{U}$ and the line $\alpha+\beta=1$ is $\frac{1}{4}$. Considering the value range of $\alpha$ and using the discriminant of quartic equation, we know that when $b \in\left[\frac{3-2 \sqrt{2}}{2}, \frac{1}{2}\right)$, Eq. (9) has no solution; and when $b \in\left[0, \frac{3-2 \sqrt{2}}{2}\right)$, Eq. (9) has only one solution given by

$$
\alpha_{U}=\frac{1-2 \sqrt{b}-\sqrt{1-4 b^{2}-4 \sqrt{b}(1-2 b)}}{2(1-2 b)} .
$$

Substituting this solution into (8) gives the corresponding ordinate of the intersection

$$
\beta_{U}=\frac{1-4 b+2 \sqrt{b}-\sqrt{1-4 b^{2}-4 \sqrt{b}(1-2 b)}}{2(1-2 b)} .
$$

Symmetrically, the intersection coordinate of the lower boundary curves of $B_{1 L}$ and $R_{L}$ is given by

$$
\begin{aligned}
\alpha_{L} & =\frac{1-4 b+2 \sqrt{b}-\sqrt{1-4 b^{2}-4 \sqrt{b}(1-2 b)}}{2(1-2 b)}, \\
\beta_{L} & =\frac{1-2 \sqrt{b}-\sqrt{1-4 b^{2}-4 \sqrt{b}(1-2 b)}}{2(1-2 b)} .
\end{aligned}
$$

Now, we are ready to prove the following theorem. 
Theorem 2 The region $R$ is a globally attractive invariant set in the state space of system (4).

Proof By Corollary 1 we know that for any initial state $\left(\alpha_{0}, \beta_{0}\right) \in U \cup L$, there must exists a positive integer $k^{*}$ such that the trajectory of system (4) starting from $\left(\alpha_{0}, \beta_{0}\right)$ enters the region $B_{1}$ after $k^{*}$-step iteration, i.e., $\left(\alpha_{k^{*}}, \beta_{k^{*}}\right) \in B_{1}$. Then, by Proposition 5 , we have $\left(\alpha_{k^{*}+1}, \beta_{k^{*}+1}\right) \in R$. The rest of this proof is to show that $R$ is an invariant set, i.e., the system trajectory will remain in $R$ for all $k>k^{*}$.

By Proposition 6 we know $R \subset B_{1} \cup B_{2}$. If $R \cap B_{2}=\emptyset$ i.e., $R \subset B_{1}$, using Proposition 5 again we have $\left(\alpha_{k^{*}+2}, \beta_{k^{*}+2}\right) \in R$ and so on. The theorem is thus true in this case (see Fig. 5 for illustration). Now, we just need to consider the rest case: $R \bigcap B_{2} \neq \emptyset$. For this case we just need to prove that once $\left(\alpha_{k^{*}}, \beta_{k^{*}}\right) \in R_{U} \bigcap B_{2 U}$, its next step $\left(\alpha_{k^{*}+1}, \beta_{k^{*}+1}\right)$ will not fall in the region $B_{1 U} \backslash R_{U}$.

By Proposition 7, $R \bigcap B_{2} \neq \emptyset$ implies that there is only one intersection point between two boundary curves, a part of the region $R$ is inside $B_{2}$, and the other part of $R$ is outside $B_{1}$ (see Fig. 6 for illustration). Since $R \subset B_{1} \cup B_{2}$ by Proposition 6 , to ensure that $R$ is an invariant set in this case it suffices to prove the following fact: once the system state falls in the region $R_{U} \bigcap B_{2 U}$, its next step will not enter the region $B_{1 U} \backslash R_{U}$. This fact is indeed true because the region $B_{1 U} \backslash R_{U}$ is on the left of the region $B_{2 U} \cap R_{U}$ (i.e., the abscissa of any point in $B_{1 U} \backslash R_{U}$ is less than the abscissa of any point in $\left.B_{2 U} \cap R_{U}\right)$, and $\alpha_{k+1}=1-\left(1-F\left(b, \alpha_{k}\right)\right)^{2} \geq F\left(b, \alpha_{k}\right) \geq \alpha_{k}$ holds for all $\left(\alpha_{k}, \beta_{k}\right)$ in region $U$ in which $\alpha_{k} \leq \frac{1}{2}$.

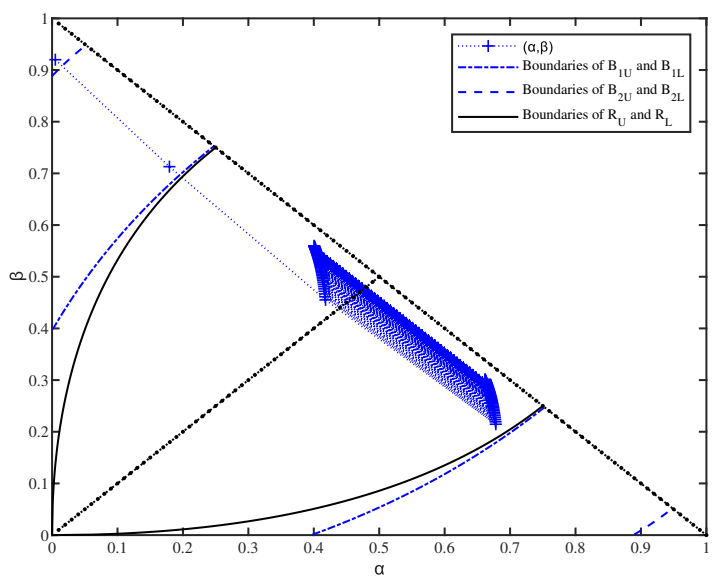

Fig. 5 Regions $B_{1}, B_{2}, R$ and iterative trajectory when $b=0.09$.

The following theorem characterizes the dynamic property of the system trajectory in the invariant set $R$.

Theorem 3 If $b \in\left[\frac{3-2 \sqrt{2}}{2}, \frac{1}{2}\right)$, then $R \subset B_{1}$, and system (4) in $R$ is in a switching iterative mode with a fixed beat number of 1 ; if $b \in\left[0, \frac{3-2 \sqrt{2}}{2}\right)$, then $R \subset B_{1} \cup B_{2}$, 


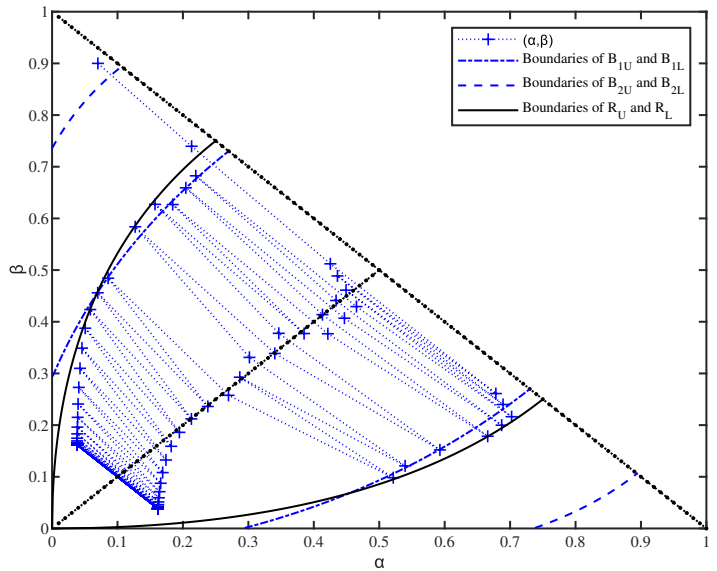

Fig. 6 Regions $B_{1}, B_{2}, R$ and iterative trajectory when $b=0.05$.

and system (4) in $R$ is in a switching iteration mode with the maximum beat number of 2.

Proof The proof is obvious based on Propositions 6, 7 and Theorem 2.

Fig. 5 presents a system trajectory for the case of $b=0.09>\frac{3-2 \sqrt{2}}{2}$, and Fig. 6 presents a system trajectory for the case of $b=0.05<\frac{3-2 \sqrt{2}}{2}$. The switching modes of the system for the case of $b=0.09$ and for the case of $b=0.05$ are presented in Fig. 7 and Fig. 8, respectively.



Fig. 7 Iteration mode when $b>\frac{3-2 \sqrt{2}}{2}$. 


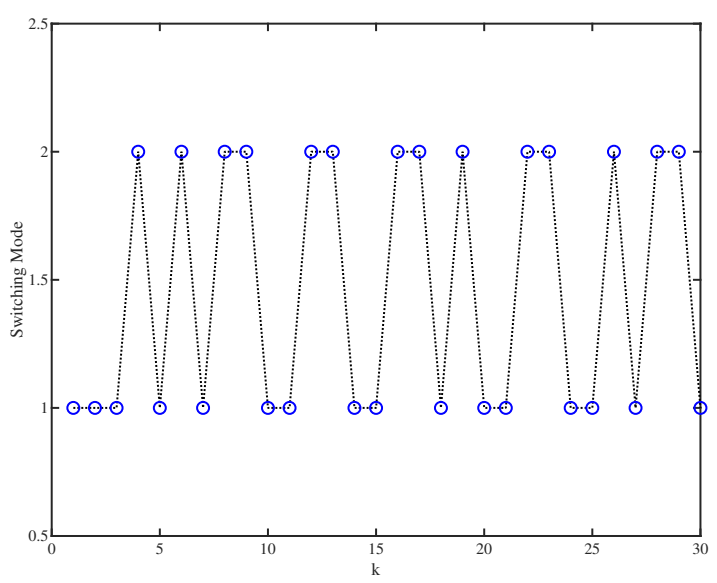

Fig. 8 Iteration mode when $b<\frac{3-2 \sqrt{2}}{2}$.

\section{Asymptotic Property of Total Error Probability}

The total error probability $L_{k}$ at layer $k$ of the tree network is the sum of false-alarm probability and missed-detection probability at the same layer, i.e., $L_{k}=\alpha_{k}+\beta_{k}$. The total error probability is a very important performance index of detection network. Since the false-alarm probability and the missed-detection probability usually do not increase or decrease simultaneously, the asymptotic property of the total error probability with the increase of the detection network size is an important topic worthy of attention.

Proposition 8 Consider dynamic system (4). $L_{k+1}(<,>,=) L_{k}$ is equivalent to

$$
\begin{cases}\alpha_{k}(<,>,=) \beta_{k}-\frac{2}{(1-2 b)^{2}}, & \text { if } \alpha_{k} \leq \beta_{k} \\ \beta_{k}(<,>,=) \alpha_{k}-\frac{2 b}{(1-2 b)^{2}}, & \text { if } \alpha_{k}>\beta_{k}\end{cases}
$$

Proof We just give the proof for the " $>$ " part because the proof for " $<$ " and "=" is the same. Moreover, because of the dynamic symmetry of the system, we just need to consider the case of $\alpha_{k} \leq \beta_{k}$.

Since $L_{k}=\alpha_{k}+\beta_{k}$ and $L_{k+1}=1-\left(1-F\left(b, \alpha_{k}\right)\right)^{2}+\left(F\left(b, \beta_{k}\right)\right)^{2}$, we have

$$
\begin{aligned}
L_{k+1}>L_{k} & \Leftrightarrow 1-\left(1-F\left(b, \alpha_{k}\right)\right)^{2}+\left(F\left(b, \beta_{k}\right)\right)^{2}>\alpha_{k}+\beta_{k}, \\
& \Leftrightarrow\left(F\left(b, \beta_{k}\right)+F\left(b, \alpha_{k}\right)-1\right)\left(F\left(b, \beta_{k}\right)-F\left(b, \alpha_{k}\right)+1\right)>\alpha_{k}+\beta_{k}-1 .
\end{aligned}
$$

Since $F\left(b, \beta_{k}\right)+F\left(b, \alpha_{k}\right)-1 \leq 0, L_{k+1}>L_{k}$ is equivalent to

$$
F\left(b, \beta_{k}\right)-F\left(b, \alpha_{k}\right)<\frac{\alpha_{k}+\beta_{k}-1}{F\left(b, \alpha_{k}\right)+F\left(b, \beta_{k}\right)-1}-1=\frac{2 b}{1-2 b} .
$$

By Eq. (1), the above inequality is also equivalent to

$$
\beta_{k}-\alpha_{k}<\frac{2 b}{(1-2 b)^{2}} \text {. }
$$


The proposition is thus proved.

Now, let us define

$$
\begin{aligned}
M_{U} & :=\left\{(\alpha, \beta) \in U \mid \beta-\alpha<\frac{2 b}{(1-2 b)^{2}}\right\}, \\
M_{L} & :=\left\{(\alpha, \beta) \in L \mid \alpha-\beta<\frac{2 b}{(1-2 b)^{2}}\right\}, \\
\partial M_{U} & :=\left\{(\alpha, \beta) \in U \mid \beta-\alpha=\frac{2 b}{(1-2 b)^{2}}\right\}, \\
\partial M_{L} & :=\left\{(\alpha, \beta) \in L \mid \alpha-\beta=\frac{2 b}{(1-2 b)^{2}}\right\},
\end{aligned}
$$

and denote $M=M_{U} \cup M_{L}, \partial M=\partial M_{U} \cup \partial M_{L}$. Obviously, $\bar{M}=M \cup \partial M$.

Based on these definitions and Proposition 8, the proof of the following theorem is obvious.

Theorem 4 Consider dynamic system (4). If $\left(\alpha_{k}, \beta_{k}\right) \in M$, then $L_{k}<L_{k+1}$; if $\left(\alpha_{k}, \beta_{k}\right) \in$ $\partial M$, then $L_{k}=L_{k+1}$; if $\left(\alpha_{k}, \beta_{k}\right) \notin \bar{M}$, then $L_{k}>L_{k+1}$.

Theorem 4 provides a necessary and sufficient condition of the convergence of the total error probability. From Theorems 2 and 4, it is natural to draw the following corollary.

Corollary 2 If $b \neq 0$, then $L_{k}$ does not approach to zero as $k \rightarrow \infty$.

Proof We prove this corollary by contradiction. Suppose that $L_{k} \rightarrow 0$ as $k \rightarrow \infty$. By Theorem 2 we know that $\left(\alpha_{k}, \beta_{k}\right)$ will remain in the invariant set $R$ when $k$ is large enough. If $L_{k} \rightarrow 0$, then $\alpha_{k} \rightarrow 0$ and $\beta_{k} \rightarrow 0$. This implies that $\left(\alpha_{k}, \beta_{k}\right)$ will enter a sufficiently small neighborhood of $(0,0)$ as $k$ becomes large enough. Noticing that $R$ is not related to $b$ we know that the intersection of $R$ with this neighborhood must belong to $M$ as long as $b \neq 0$. By Theorem 4 , once $\left(\alpha_{k}, \beta_{k}\right)$ enters $M, L_{k+1}>L_{k}$ always holds. This contradicts the hypothesis $L_{k} \rightarrow 0$.

Although $(0,0)$ is an equilibrium point of dynamic system (4), Corollary 2 tells us that when there are bit errors in communication channels of the tree detection network, it is impossible to make the total error probability of the fusion center tend to zero by increasing the scale of the network.

Since $M$ is related to $b$ but $R$ is not, it can be expected that when $b$ is large enough, the entire invariant set $R$ may fall into $M$, which means that when $k$ is large enough, the total error probability $L_{k}$ is continuously increasing. Obviously, this is a situation that should be avoided in network design. In addition, since $B_{1}$ is the only mode-switching region in the state space, clarifying the relationship between region $M$ and region $B_{1}$ is helpful to analyze the iterative mode of the dynamic system when $\left(\alpha_{k}, \beta_{k}\right)$ falls into the non-decreasing region $\bar{M}$ for $L_{k}$.

Theorem 5 The following statements are true for dynamic system (4). 
(1) If $b \in\left[0, \frac{2-\sqrt{3}}{2}\right)$, then $R \backslash M \neq \emptyset, M \backslash R \neq \emptyset$, and $\bar{M} \subset B_{1}$.

(2) If $b \in\left[\frac{2-\sqrt{3}}{2}, \frac{1}{2}\right)$, then $R \subset M$. In particular, if $b=\frac{2-\sqrt{3}}{2}$, the upper boundary of $\overline{M_{U}}$ and the upper boundary of $R_{U}$ have only one intersection point, and the lower boundary of $\overline{M_{L}}$ and the lower boundary of $R_{L}$ also have only one intersection point, both upper and lower intersections are located on the line $\alpha+\beta=1$.

Proof of Theorem 5 is given in Appendix E.

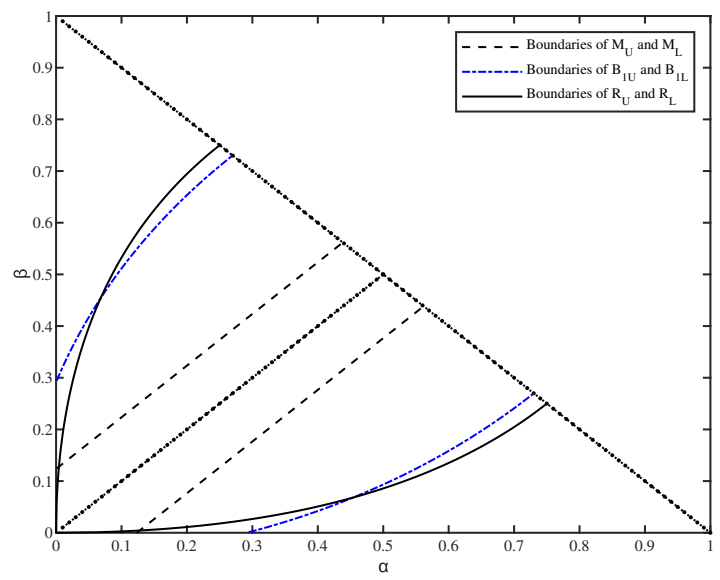

Fig. 9 Region $M, B_{1}$ and $R$ when $b<\frac{2-\sqrt{3}}{2}$.

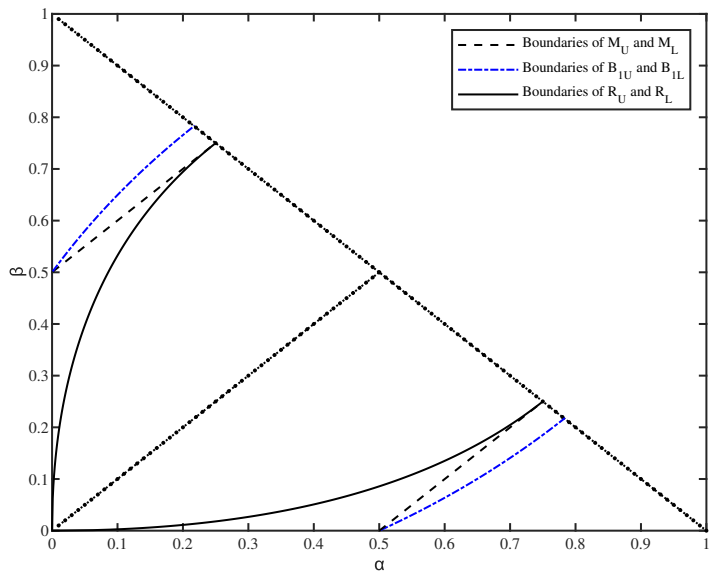

Fig. 10 Region $M, B_{1}$ and $R$ when $b=\frac{2-\sqrt{3}}{2}$.

Fig. 9 shows the relationship of $M, B_{1}$ and $R$ when $b=0.05$. Since $b=0.05<$ $\frac{2-\sqrt{3}}{2}$, according to Theorem $5, R \backslash M \neq \emptyset, M \backslash R \neq \emptyset$, and $\bar{M} \subset B_{1}$. Fig. 10 shows 
the relationship of $M, B_{1}$ and $R$ when $b=\frac{2-\sqrt{3}}{2}$. In this case, according to Theorem $5, R \subset \bar{M}$, the upper boundary of $\overline{M_{U}}$ and the upper boundary of $R_{U}$ have only one intersection point, the lower boundary of $\overline{M_{L}}$ and the lower boundary of $R_{L}$ also have only one intersection point.

According to Theorem 2, Theorem 4 and Theorem 5, the following corollary is obvious.

Corollary 3 Consider dynamic system (4). If $b \in\left[\frac{2-\sqrt{3}}{2}, \frac{1}{2}\right)$, then for any initial state $\left(\alpha_{0}, \beta_{0}\right)$, there must exist a positive integer $k^{*}$ such that the total error probability $L_{k}$ will continuously increasing when $k \geq k^{*}$.

In order to avoid the situation mentioned in Corollary 3, in the design of wireless sensor networks, the bit error probability must be kept in the range $b<\frac{2-\sqrt{3}}{2}$.



Fig. 11 Iteration of $L_{k}$ for the case $b<\frac{2-\sqrt{3}}{2}$ and the case $b>\frac{2-\sqrt{3}}{2}$.

Fig. 11 presents simulation results for $b=0.05<\frac{2-\sqrt{3}}{2}$ and $b=0.25>\frac{2-\sqrt{3}}{2}$. In the former case, $L_{k}$ decreases to some non-zero constant; but in the latter case, $L_{k}$ continuously increases to 1 as $k \rightarrow \infty$.

\section{Conclusion}

In this paper, the evolution of detection performance of balanced binary tree networks with channel noise is studied. Firstly, the iterative rules of false-alarm probability and missed detection probability are modeled as a state-dependent nonlinear switched system. Then, according to the channel bit error probability $b$, the system state space is divided into two parts: the mode-switching region and the mode-holding region. The iterative trajectory of the system state is described, and a globally attractive invariant set which is independent of the channel bit error probability is found. The necessary and sufficient condition of the convergence of the total error probability of 
the detection network is obtained. Through our analysis, the following important conclusions are obtained: (1) as long as the channel bit error probability is not zero, the total error probability of the detection network will not tend to zero with the increase of network size, which is essentially different from the conclusion obtained when the network has nodes and link failures [13]. (2) When the channel bit error probability is greater than $\frac{2-\sqrt{3}}{2}$, the total error probability continuously increases with the increase of network size. These results provide a theoretical basis for the design and performance analysis of large-scale detection networks.

Some interesting questions remain open. Firstly, in the case of $b \in\left[0, \frac{2-\sqrt{3}}{2}\right)$, since $R \backslash M \neq \emptyset$ and $M \backslash R \neq \emptyset$ (see Theorem 5), it is currently not clear wether the total error probability converges to a positive constant. Secondly, for the researchers of nonlinear dynamics, the unknown (possibly aperiodic) switching law of the system in the case of $b \in\left[0, \frac{3-2 \sqrt{2}}{2}\right)$ (see Theorem 3 and Fig. 8) may seem attractive. Finally, all our results are obtained under the assumption that the sensors make independent detection observations and have identical detection property, which is of course restrictive. The asymptotic detection performance of the network with correlated sensors is worth of further study.

Acknowledgements This work was supported by the National Natural Science Foundation of China under Grant 62073107 and Natural Science Foundation of Zhejiang Province under Grant LZ21F030002.

\section{Conflict of interest}

The authors declare that they have no conflict of interest.

\section{Appendices}

\section{A Proof of Proposition 2}

We only need to prove $\left.\left.B_{1 U}\right|_{b=0} \subset B_{1 U}\right|_{b \neq 0}$ due to the dynamic symmetry of the system.

By the definition of $B_{1 U}$, the upper boundary of region $B_{1 U}$ is described by equation of $\left(1-(F(b, \alpha))^{2}+\right.$ $(F(b, \beta))^{2}=1$. For any constant abscissa $\alpha$, this equation define an implicit function $\beta(b)$, where $b \in$ $\left[0, \frac{1}{2}\right)$. To prove the proposition, it suffices to prove the positivity of the derivative of this function, i.e., $\beta_{b}^{\prime}=\frac{\mathrm{d} \beta}{\mathrm{d} b}>0$. By derivation rules of implicit functions it is easy to get

$$
\beta_{b}^{\prime}=\frac{(1-F(b, \alpha))(1-2 \alpha)-F(b, \beta)(1-2 \beta)}{F(b, \beta)(1-2 b)} .
$$

Since $\beta>\alpha$ due to $(\alpha, \beta) \in U$, we have

$$
\beta_{b}^{\prime}>\frac{(1-2 \alpha)(1-F(b, \alpha)-F(b, \beta))}{F(b, \beta)(1-2 b)} .
$$

Now, let us check the intersection point of the upper boundary of $B_{1 U}$ with the line $\alpha+\beta=1$. Combining their equations yields $[(1-2 b) \beta+b]^{2}=\frac{1}{2}$, which implies that $\beta=\frac{\left(\frac{1}{2}\right)^{\frac{1}{2}}-b}{1-2 b} \geq\left(\frac{1}{2}\right)^{\frac{1}{2}}$, or equivalently, $\alpha \leq 1-\left(\frac{1}{2}\right)^{\frac{1}{2}}$. It implies that the abscissa of the intersection point of the upper boundary of $B_{1 U}$ with the line $\alpha+\beta=1$ is less than $\frac{1}{2}$. Considering the fact that $1-2 b>0$ by Assumption 2 and $1-F(b, \alpha)-F(b, \beta) \geq 0$ by (3), we get $\beta_{b}^{\prime}>0$. The proposition is thus proved. 


\section{B Proof of Proposition 3}

Suppose that $\left(\alpha_{k}, \beta_{k}\right) \in B_{m U}, m \geq 2$. By definition of $B_{m U}$, we get that

$$
D^{m-1}\left(b,\left(1-F\left(b, \alpha_{k}\right)\right)+D^{m-1}\left(b, F\left(b, \beta_{k}\right)\right)<1,\right.
$$

and

$$
D^{m-2}\left(b,\left(1-F\left(b, \alpha_{k}\right)\right)+D^{m-2}\left(b, F\left(b, \beta_{k}\right)\right) \geq 1 .\right.
$$

Note that here we let $D^{0}(b, y)=y^{2}$ for simplicity of notation.

By (4), it is easy to get $F\left(b, \alpha_{k+1}\right)=1-b-(1-2 b)\left(1-F\left(b, \alpha_{k}\right)\right)^{2}, F\left(b, \beta_{k+1}\right)=(1-2 b)\left(F\left(b, \beta_{k}\right)\right)^{2}+$ $b$. Hence, from (10) and (11) it follows that

$$
D^{m-2}\left(b,\left(1-F\left(b, \alpha_{k+1}\right)\right)+D^{m-2}\left(b, F\left(b, \beta_{k+1}\right)\right)<1,\right.
$$

and

$$
D^{m-3}\left(b,\left(1-F\left(b, \alpha_{k+1}\right)\right)+D^{m-3}\left(b, F\left(b, \beta_{k+1}\right)\right) \geq 1 .\right.
$$

Note that here we assume $D^{-1}(b, F(b, x))=x$ and $D^{-1}(b,(1-F(b, x)))=1-x$ for simplicity of notation. The above two inequalities imply that $\left(\alpha_{k+1}, \beta_{k+1}\right) \in B_{(m-1) U}$. Due to the dynamic symmetry of the system, the case of $\left(\alpha_{k}, \beta_{k}\right) \in B_{m L}$ can be proved similarly.

\section{Proof of Proposition 4}

Due to the dynamic symmetry of the system, we just need to prove $U \subseteq \cup_{m=1}^{\infty} B_{m} U$

Let us check the intersection point of the upper boundary of $B_{m U}$ with the line $\alpha+\beta=1$. The upper boundary equation of $B_{m U}$ is given by

$$
D^{m-1}\left(b,(1-F(b, \alpha))+D^{m-1}(b, F(b, \beta))=1 .\right.
$$

Combining this equation and the line equation $\alpha+\beta=1$ we get

$$
D^{m-1}(b, F(b, \beta))=\frac{1}{2} .
$$

In Proof of Proposition 2 we have shown that for $m=1$, this equation implies $\beta=\frac{\left(\frac{1}{2}\right)^{\frac{1}{2}}-b}{1-2 b} \geq\left(\frac{1}{2}\right)^{\frac{1}{2}}$. Using mathematical induction, we can show that Eq. (12) implies

$$
\beta \geq \lim _{m \rightarrow \infty} \frac{\left(\frac{1}{2}\right)^{\frac{1}{2 m}}-b}{1-2 b} \geq \lim _{m \rightarrow \infty}\left(\frac{1}{2}\right)^{\frac{1}{2 m}}=1,
$$

which indicates that the ordinate of the intersection point of the upper boundary of $B_{m U}$ with line $\alpha+\beta=1$ is not less than 1 as $m \rightarrow \infty$. This also implies that the ordinate of the intersection point of the upper boundary of $B_{m U}$ with the axis $\alpha=0$ is not less than 1 . Since the convexity of the upper boundary curve of $B_{m U}$ is obvious, the upper triangular of the state space of the system is below this boundary as $m \rightarrow \infty$, i.e., $U \subseteq \bigcup_{m=1}^{\infty} B_{m U}$. Proposition 4 is thus proved.

\section{Proof of Proposition 6}

Due to the dynamic symmetry of the system, we just need to prove $R_{U} \subset B_{1 U} \cup B_{2 U}$, i.e, to prove the upper boundary of $B_{2 U}$ is always higher than the upper boundary of $R_{U}$. Our proof will use the following lemma which is proved in [12].

Lemma $1 R_{U} \subset B_{1 U} \cup B_{2 U}$ holds for $b=0$

Since $R_{U}$ is independent of $b$, Lemma 1 tells us that to prove Proposition 6 it suffices to prove that the upper boundary of $B_{2 U}$ with $b \neq 0$ is higher than the upper boundary of $B_{2 U}$ with $b=0$. 
Suppose that $\left(\alpha_{k}^{0}, \beta_{k}^{0}\right)$ is a point on the upper boundary of $B_{2 U}$ with $b=0$, and $\left(\alpha_{k}^{b}, \beta_{k}^{b}\right)$ is a point on the upper boundary of $B_{2 U}$ with $b \in\left(0, \frac{1}{2}\right)$. To prove Proposition 6 , it suffices to prove that $\beta_{k}^{b}>\beta_{k}^{0}$ when $\alpha_{k}^{0}=\alpha_{k}^{b}$.

For any point $\left(\alpha_{k}^{b}, \beta_{k}^{b}\right)$ on the upper boundary of $B_{2 U}$, since $\left[(1-2 b)\left(1-F\left(b, \alpha_{k}^{b}\right)\right)^{2}+b\right]^{2}+[(1-$ $\left.2 b)\left(F\left(b, \beta_{k}^{b}\right)\right)^{2}+b\right]^{2}=1$, using Eq. (4) we get $\left(1-F\left(b, \alpha_{k+1}^{b}\right)\right)^{2}+\left(F\left(b, \beta_{k+1}^{b}\right)\right)^{2}=1$. This implies that $\left(\alpha_{k+1}^{b}, \beta_{k+1}^{b}\right)$ lies on the upper boundary of $B_{1 U}$. For $b=0$, the upper boundary equation of the region $B_{1 U}$ is $(1-\alpha)^{2}+\beta^{2}=1$. Based on this boundary equation we define a function $\beta=G_{0}(\alpha)$. Obviously, $G_{0}(\alpha)$ is monotony increasing. For $b \in\left(0, \frac{1}{2}\right)$, the upper boundary equation of the region $B_{1 U}$ is $(1-F(b, \alpha))^{2}+$ $F(b, \beta)^{2}=1$. Based on this boundary equation we define another function $\beta=G_{b}(\alpha)$. Therefore, for $\left(\alpha_{k}^{0}, \beta_{k}^{0}\right)$ on the upper boundary of $B_{2 U}$ with $b=0$ and $\left(\alpha_{k}^{b}, \beta_{k}^{b}\right)$ on the upper boundary of $B_{2 U}$ with $b \in\left(0, \frac{1}{2}\right)$, we know that $\beta_{k+1}^{0}=G_{0}\left(\alpha_{k+1}^{0}\right)$ and $\beta_{k+1}^{b}=G_{b}\left(\alpha_{k+1}^{b}\right)$.

Since $F(b, \alpha)>\alpha$ holds for any $b \in\left(0, \frac{1}{2}\right)$ and $\alpha_{k}^{0}=\alpha_{k}^{b}$ as supposed, we have $F\left(b, \alpha_{k}^{b}\right)>\alpha_{k}^{0}$. Therefore,

Then, by Proposition 2 we have

$$
\left\{\begin{array}{l}
\alpha_{k+1}^{0}=1-\left(1-\alpha_{k}^{0}\right)^{2} \\
\alpha_{k+1}^{b}=1-\left(1-F\left(b, \alpha_{k}^{b}\right)\right)^{2}
\end{array} \Rightarrow \alpha_{k+1}^{b}>\alpha_{k+1}^{0} .\right.
$$

$$
G_{b}\left(\alpha_{k+1}^{b}\right)>G_{0}\left(\alpha_{k+1}^{b}\right)>G_{0}\left(\alpha_{k+1}^{0}\right) .
$$

The above discussion has shown that $\beta_{k+1}^{b}>\beta_{k+1}^{0}$ under the condition $\alpha_{k}^{0}=\alpha_{k}^{b}$, Therefore, from the iteration formula $\beta_{k+1}=\left(F\left(b, \beta_{k}\right)\right)^{2}$ it follows that

$$
\left\{\begin{array}{l}
\beta_{k+1}^{0}=\left(\beta_{k}^{0}\right)^{2} \\
\beta_{k+1}^{b}=\left(F\left(b, \beta_{k}^{b}\right)\right)^{2}
\end{array} \Rightarrow F\left(b, \beta_{k}^{b}\right)>\beta_{k}^{0} \Rightarrow \beta_{k}^{b}>\frac{\beta_{k}^{0}-b}{1-2 b} .\right.
$$

Similar to what we have shown in Proof of Proposition 4, the ordinate of the intersection point of the upper boundary with the line $\alpha+\beta=1$ is greater than $1 / 2$. Hence,

$$
\frac{\mathrm{d}\left(\frac{\beta_{k}^{0}-b}{1-2 b}\right)}{\mathrm{d} b}=\frac{2 \beta_{k}^{0}-1}{(1-2 b)^{2}}>0
$$

for all $\beta_{k}^{0} \in[0.5,1]$, which implies that

$$
\inf _{b \in(0,0.5)}\left(\frac{\beta_{k}^{0}-b}{1-2 b}\right)=\beta_{k}^{0}
$$

for all $\beta_{k}^{0} \in[0.5,1]$. Thus, $\beta_{k}^{b}>\beta_{k}^{0}$. Proposition 6 is proved.

\section{E Proof of Theorem 5}

By the dynamic symmetry of the system, the proof is just provided for the region $U$.

Firstly, we prove the part on the relationship of $M_{U}$ and $R_{U}$. The upper boundary equation of $M_{U}$ is $\beta=\alpha+\frac{2 b}{(1-2 b)^{2}}$, and the upper boundary equation of $R_{U}$ is $\beta=2 \sqrt{\alpha}-\alpha$. Let us define function

$$
\begin{aligned}
P(\alpha) & =\alpha+\frac{2 b}{(1-2 b)^{2}}-(2 \sqrt{\alpha}-\alpha) \\
& =2\left[\frac{b}{(1-2 b)^{2}}-\sqrt{\alpha}+\alpha\right]
\end{aligned}
$$

for $\alpha \in\left[0, \frac{1}{4}\right]$. The derivative of this function is $P^{\prime}(\alpha)=2-\frac{1}{\sqrt{\alpha}} \leq 0$. Therefore, $P_{\max }(\alpha)=P(0) \geq$ $0, P_{\min }(\alpha)=P\left(\frac{1}{4}\right)$, where

$$
\left\{\begin{array}{l}
P\left(\frac{1}{4}\right)<0, \text { if } b \in\left[0, \frac{2-\sqrt{3}}{2}\right), \\
P\left(\frac{1}{4}\right)=0, \text { if } b=\frac{2-\sqrt{3}}{2} \\
P\left(\frac{1}{4}\right)>0, \text { if } b \in\left(\frac{2-\sqrt{3}}{2}, \frac{1}{2}\right) .
\end{array}\right.
$$


Therefore, when $b \in\left[0, \frac{2-\sqrt{3}}{2}\right), R_{U} \backslash M_{U} \neq \emptyset, M_{U} \backslash R_{U} \neq \emptyset$ hold; when $b=\frac{2-\sqrt{3}}{2}, R_{U} \subset \overline{M_{U}}$ holds, and the upper boundary of $M_{U}$ and the upper boundary of $R_{U}$ have only one intersection point $\left(\frac{1}{4}, \frac{3}{4}\right)$; when $b \in\left(\frac{2-\sqrt{3}}{2}, \frac{1}{2}\right), R_{U} \subset M_{U}$ holds, and and the upper boundary of $M_{U}$ and the upper boundary of $R_{U}$ have no intersection.

Next, we prove the part on the relationship of $M_{U}$ and $B_{1 U}$.

The upper boundary equation of $M_{U}$ can be also written as $F(b, \beta)=F(b, \alpha)+\frac{2 b}{1-2 b}$, and the upper boundary equation of $B_{1 U}$ is $F(b, \beta)=\sqrt{2 F(b, \alpha)-(F(b, \alpha))^{2}}$. Let us introduce a variable $t=F(b, \beta)$ and define function

$$
Q(y)=y+\frac{2 b}{1-2 b}-\sqrt{2 y-y^{2}}
$$

for $y \in\left[b, \frac{2-\sqrt{2}}{2}\right]$. The derivative of this function is $Q^{\prime}(y)=1-\frac{1-y}{\sqrt{2 y-y^{2}}} \leq 0$. Therefore,

$$
Q_{\max }(y)=Q(b)=b+\frac{2 b}{1-2 b}-\sqrt{2 b-b^{2}} .
$$

Obviously, when $b \in\left[0, \frac{2-\sqrt{3}}{2}\right), Q_{\max }(y) \leq 0$. Hence, the upper boundary of $B_{1 U}$ must be higher than the upper boundary of $M_{U}$. The proof is completed.

\section{References}

1. Tenney R. R., Sandell N. R.: Detection with distributed sensors. IEEE Trans. Aerosp. Electron. Syst. AES-17(4), 501-510(1981)

2. Chair Z., Varshney P.: Optimal data fusion in multiple sensor detection systems. IEEE Trans. Aerosp. Electron. Syst. 22(1), 98-101(1986)

3. Tsitsiklis J. N.: Decentralized detection. Adv. Statist. Signal Process. 2(2), 297-344(1993)

4. Varshney P. K.: Distributed Detection and Data Fusion. Springer-Verlag, New York (1997)

5. Banavar M. K., Smith A. D., Tepedelenlioglu C., Spanias A.: On the effectiveness of multiple antennas in distributed detection over fading MACs. IEEE Trans. Wireless Commun. 11(5), 1744-1752(2012)

6. Tang Z. B., Pattipati K. R., Kleinman D. L.: Optimization of detection networks: Part IłTandem structures. IEEE Trans. Syst., Man, Cybern. 21(5), 1044-1059(1991)

7. Tay W. P., Tsitsiklis J. N., Win M. Z.: On the subexponential decay of detection error probabilities in long tandems. IEEE Trans. Inf. Theory 54(10), 4767-C4771(2008)

8. Ho J., Tay W. P., Quek T. Q. S., Chong E. K. P.: Robust decentralized detection and social learning in tandem networks. IEEE Trans. Signal Process. 63(19), 5019-C5032(2015)

9. Tay W. P., Tsitsiklis J. N., Win M. Z., Bayesian detection in bounded height tree networks. IEEE Trans. Signal Process. 57(10), 4042-C4051(2009)

10. Gubner J. A., Chong E. K. P., Scharf L. L.: Aggregation and compression of distributed binary decisions in a wireless sensor network. In: The 48th IEEE Conference on Decision and Control, Shanghai, China, pp.909-913(2009)

11. Zhang Z., Chong E. K. P., Pezeshki A., Moran B., Howard S. D.: Near-optimal distributed detection in balanced binary relay trees. IEEE Trans. Contr. Net. Syst. 4(4), 826-837(2017)

12. Zhang Z., Pezeshki A., Moran W., Howard S. D., Chong E. K. P.: Error probability bounds for balanced binary relay trees. IEEE Trans. Inf. Theory 58(6), 3548-3563(2012)

13. Zhang Z., Chong E. K. P., Pezeshki A., Moran W., Howard S. D.: Detection performance in balanced binary relay trees with node and link failures. IEEE Transactions on Signal Processing 61(9), 21652177(2013)

14. Tian Y.-P., Sun X.-J., Tian O.: Detection performance of the majority dominance rule in m-ary relay trees with node and link failures. IEEE Trans. Signal Process. 66(6), 1469-1482(2018)

15. Jayaweera S. K.: Large sensor system performance of decentralized detection in noisy, bandlimited channels, In: The 61st IEEE Vehicular Technology Conference, Stockholm, Sweden, pp. 10961100(2005)

16. Kashyap A.: Comments on On the optimality of the likelihood-ratio test for local sensor decision rules in the presence of nonideal channels. IEEE Trans. Inf. Theory 52(3), 1274-1275(2006) 
17. Kar S., Moura J. M. F.: Distributed consensus algorithms in sensor networks with imperfect communication: Link failures and channel noise. IEEE Trans. Signal Process. 57(1), 355-369(2009)

18. Liu G., Xu B., Zeng M., Chen H.: Distributed estimation over binary symmetric channels in wireless sensor networks. IET Wireless Sensor Systems 1(2), 105-109(2011)

19. Nardelli P. H. J., Ramezanipour I., Alves H., de Lima C. H. M., Latva-Aho M.: Average error probability in wireless sensor networks with imperfect sensing and communication for different decision rules. IEEE Sensors Journal 16(10), 3948-3957(2016)

20. Cheng X., Ciuonzo D., Rossi P. S.: Multibit decentralized detection through fusing smart and dumb sensors based on Rao test. IEEE Trans. Aerosp. Electron. Syst. 56(2), 1391-1405(2020)

21. Aysal T. C., Barner K. E.: Decentralized estimation over noisy channels for bandwidth-constrained sensor networks. In: The 2007 IEEE International Conference on Acoustics, Speech and Signal Processing, Honolulu, HI, pp.929-932(2007)

22. Huang Y., Tian Y.-P.: Detection Performance in m-ary relay trees with link failures and unreliable communications. In: The 15th IEEE International Conference on Control and Automation, Edinburgh, UK, pp.1575-1580(2019) 